\title{
Value of antenatal diagnosis of abnormalities of the urinary tract
}

\author{
J D Greig, P A M Raine, D G Young, A F Azmy, J Ruth MacKenzie, F Danskin, M J Whittle, \\ M B McNay
}

Department of Paediatric Surgery, Royal Hospital for Sick Children, Glasgow G3 8SJ

J D Greig, FRCS, registrar

P A M Raine, FRCS, consultant

D G Young, FRCs, reader

A F Azmy, FRCS, consultant

Department of

Ultrasonography, Glasgow Royal Maternity Hospital, Glasgow G4 0NA

J Ruth MacKenzie, FRCR, consultant

Department of Obstetrics, Queen Mother's Hospital, Glasgow G3 8SH

F Danskin, MB, research fellow

M J Whittle, FRCOG, consultant

M B McNay, FRCOG, lecturer

Correspondence to:

Mr A F Azmy.

Br Med f 1989;298:1417-9

\begin{abstract}
Objective-To assess the value of antenatal diagnosis of abnormalities of the urinary tract on ultrasonography.

Design-Retrospective study.

Setting-Two obstetric units in Glasgow.

Subjects -62 Fetuses in which renal abnormalities were diagnosed on antenatal ultrasonography.

Interventions-Six fetuses had their bladders aspirated to determine renal function. Fifteen pregnancies were terminated on the basis of the findings on antenatal ultrasonography, and if possible necropsy was performed on the fetuses. In babies who were born alive the final diagnosis was made by postnatal ultrasonography, intravenous urography, radionuclide scanning, cystography, and, in those who died in the early neonatal period, necropsy. Neonates who were referred with a known obstructed kidney had nephrostomy or pyeloplasty.

End point-Assessment of the value of antenatal diagnosis of renal abnormalities on ultrasonography for babies who had no clinical evidence of disease postnatally.
\end{abstract}

Main results-Eighteen fetuses did not survive birth; the antenatal diagnosis was accurate in all 18. Of the $\mathbf{4 4}$ babies born alive, five had normal urinary tracts, in two of whom antenatal ultrasonography had probably indicated a false positive diagnosis. Fourteen babies died during the early neonatal period. Twenty five babies with renal abnormalities were followed up; the antenatal diagnosis was inaccurate for 10 of them, the commonest misdiagnosis being hydronephrosis for multicystic kidney and vice versa, and there was one false positive diagnosis. The initial clinical findings in 14 babies would have led to the early detection of a urological abnormality. In the $\mathbf{3 0}$ babies with no clinical evidence of disease the antenatal diagnosis was of definite value in eight, probable value in 15 , and marginal value in seven. Overall, an accurate antenatal diagnosis was made in $\mathbf{4 6}$ of the 62 cases (74\%); in 12 cases renal disease was detected but its specific nature was not determined; and in four cases the diagnosis was misleading.

Conclusions - The overall value of antenatal diagnosis is that it indicates early termination of fetuses with fatal renal disease, prepares parents and medical staff for the likelihood of serious neonatal problems, and shows abnormalities of the urinary tract that may not be detected postnatally.

\section{Introduction}

The incidence of abnormalities of the urinary tract that can be diagnosed antenatally has been estimated as 1/1000 live births. ${ }^{1}$ Such diagnoses raise new and possibly controversial questions and have implications for the fetus or neonate, or both, and for the parents and the nursing and medical team. In a study of disorders that were diagnosed antenatally by ultrasonography and required an operation we found that urological abnormalities comprised $34 \% .^{2}$ We now report cases of such abnormalities that we have encountered during the past four and a half years.

\section{Methods}

We detected 46 cases of fetal uropathy on ultrasonography in two large obstetric units in Glasgow during 54 months (August 1983 to February 1988), and 16 pregnant women were referred from other district maternity units in west Scotland after fetal uropathy was diagnosed on ultrasonography. Antenatal bladder aspirations guided by ultrasonography were performed on six fetuses to determine renal function. One fetus had a renal cyst aspirated, but no fetus received intrauterine drainage. In those pregnancies that were terminated necropsy of the fetus was performed if possible.

In babies who were born alive the final diagnosis was made by postnatal ultrasonography, intravenous urography, radionuclide scanning, cystography, and, in those who died in the early neonatal period, necropsy. Neonates who were referred with a known obstructed kidney had nephrostomy or pyeloplasty during the neonatal period. In other cases an operation was deferred after assessment or was not considered necessary. The value of antenatal diagnosis of renal abnormalities for their subsequent management was classified as marginal (for example, for mild unilateral dilatation and agenesis of one kidney); probable (for example, for unilateral obstruction of the pelviureteric junction and multicystic kidney); or definite (for example, for treatable conditions affecting both kidneys, such as posterior urethral valves).

\section{Results}

Eighteen fetuses (29\%) did not survive birth (table). Fifteen of the pregnancies were terminated on the basis of findings on antenatal ultrasonography, which had been done at a mean gestational age of 18 (range 14-23) weeks and agreed with the findings at necropsy in all cases. Eight fetuses had associated congenital abnormalities, including five with prune belly syndrome, and antenatal aspirations of the bladder confirmed poor renal function in four. Two fetuses that were not terminated (one died in utero and one was stillborn) had lethal urological abnormalities. One fetus with unilateral cystic dysplasia aborted spontaneously.

Forty four babies were born alive. Five of these had normal urinary tracts; in two of them (cases 19 and 23) ultrasonography had probably given a false positive diagnosis as follow up did not show any renal abnormalities. Three babies (cases 20-22) had had unilateral or bilateral hydronephrosis on repeated antenatal 
scanning, but this decreased towards term and at birth and consequently their upper urinary tracts were normal; we regarded these babies as having had transient intrauterine hydronephrosis, not as having yielded a false positive diagnosis.

Fourteen babies died during the early neonatal period. The abnormalities in these cases had generally been diagnosed later than those in the terminated pregnancies (mean gestational age 29 (range 17-38) weeks). If these abnormalities had not been diagnosed in utero urological problems would probably have become apparent in most of the babies after delivery. Only one fetus in this group had had a bladder aspiration. This had confirmed poor renal function at 27 weeks. The antenatal diagnoses were inaccurate in four cases, but this did not affect the outcomes. Eight of the babies had associated congenital abnormalities.

Twenty five babies with renal abnormalities were followed up. The antenatal diagnosis had been made at

\section{MINIPRINT TABLE}

Diagnosis on antenatal ulrasonography and final diagnosis in fetuses with renal abnormalities that did not
survive birth (cases $I-18$ ); in babies born with a normal renal tract (cases $19-23$ ) in babies with renal

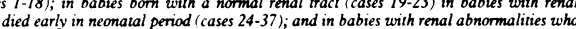
survived neonatal period. (Pregnancy was terminated in cases $1-15$ )
s.

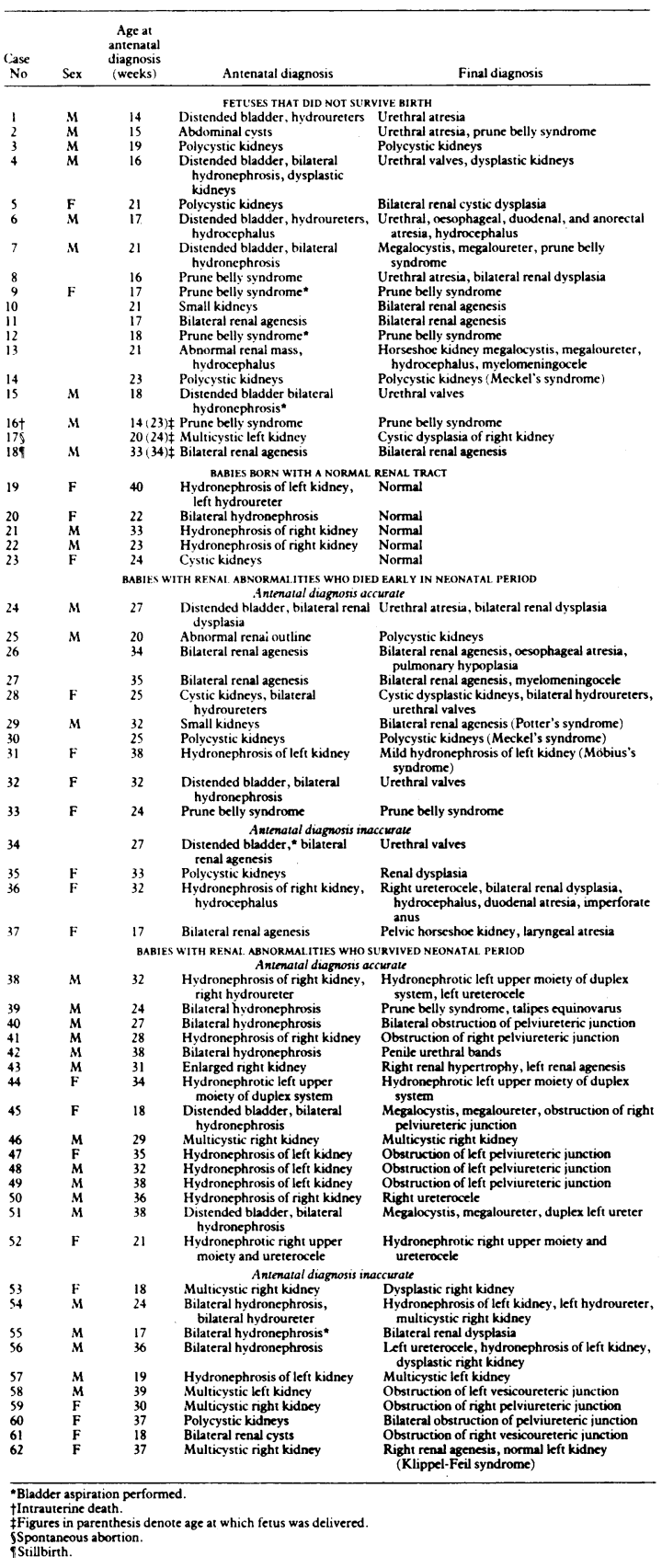

a mean gestational age of 29 (range 18-39) weeks, 18 having been diagnosed in the third trimester. The antenatal diagnosis was accurate in 15 cases but inaccurate in 10; the commonest misdiagnosis was hydronephrosis mistaken for multicystic kidney or vice versa. There was one false positive finding (case 62), when a normal left kidney (the right kidney was absent) was misdiagnosed as a multicystic right kidney.

The initial clinical findings in 14 of the 44 babies who were born alive would have led to early detection of a urological abnormality. In the 30 others, however, there was no clinical evidence of renal disease. The 30 babies were subdivided to assess the value of antenatal ultrasonography in directing the management of the renal abnormality after birth. The antenatal diagnosis was of definite value in eight of them, of probable value in 15, and of marginal value in seven (including the five babies who had normal urinary tracts after birth).

Overall, an accurate antenatal diagnosis was made in 46 of the 62 cases (74\%). In 12 cases renal disease was detected but its specific nature was not determined; and in four cases the diagnosis was misleading, but in no case did this affect the clinical outcome.

\section{Discussion}

The overall diagnostic accuracy of antenatal ultrasonography of $74 \%$ is similar to that found in comparable series. ${ }^{13}$ The main difficulty was distinguishing between dysplastic, hydronephrotic, and cystic kidneys, and this accounted for most of the inaccurate diagnoses. The diagnosis of renal agenesis is also difficult as the possibility of ectopic renal tissue has to be excluded.

No false positive diagnoses of fetal uropathy were made in any of the pregnancies that were terminated in this series, and all but two fetuses had a fatal abnormality of the urinary tract. Aspiration of the fetal bladder to confirm poor renal function was valuable and this may facilitate the decision as to whether to offer termination when the fetal abnormality is considered incompatible with life. Among the babies born alive there were three false positive diagnoses. This did not lead to adverse management in any case, though this is obviously a risk.

Fourteen (32\%) babies died during the early neonatal period. In two of these, who had posterior urethral valves, renal function might have been improved by intrauterine drainage, but there is evidence that survival is no better after such procedures, which have an unacceptably high rate of complications. ${ }^{45}$ We believe that an early operation after birth to improve the drainage of the urinary tract, particularly for obstruction of the pelviureteric junction, results in reduced parenchymal loss and preserves renal function. ${ }^{6}$ The mortality and the subsequent renal function in the babies in our series are comparable with those in other series with and without fetal intervention. ${ }^{178}$

The overall value of antenatal diagnosis in this study was that it indicated early termination of fetuses with fatal disease ( 15 cases), prepared parents and attendant nursing and medical staff for the likelihood of serious neonatal problems (there were 14 deaths in the early neonatal period), and made staff aware of abnormalities of the urinary tract in neonates without obvious clinical evidence of an abnormality postnatally (30 cases). A minority of fetuses (14) progressed to normal birth and survived with an obvious abnormality that would inevitably have been diagnosed clinically and whose management was not influenced by antenatal diagnosis. Even so, the parents' readiness for postnatal events is important psychologically and socially.

We conclude that once an abnormality of the fetal urinary tract is detected pregnant women should 
be referred to a regional centre for confirmation of the diagnosis and be monitored subsequently. The parents and medical team should discuss the management of the abnormality. Antenatal diagnosis inevitably raises moral and ethical questions, and only frequent audit of the implications and outcomes of such practices will enable clinicians to formulate the best "management policies.

1 Thomas DFM, Irvine HC, Arthur RJ. Pre-natal diagnosis: how useful is it? $\mathrm{Br} \mathcal{f}$ Urol 1985;57:784-7.

2 Hutson JM, McNay MB, MacKenzie JR, Whittle MJ, Young DG, Raine PAM.
Antenatal diagnosis of surgical disorders by ultrasonography. Lancet 1985;i:621-3.

3 Pocock RD, Witcombe JB, Andrews HS, Berry PJ, Frank JD. The outcome of antenatally diagnosed urological abnormalities. Br $\mathcal{Z}$ Urol 1985;57:788-92.

DeLuca FG. The status of prenatal diagnosis and fetai surgery. Pediatr Surg Im 1987;2:259-66

5 Elder JS, Ducken JW, Snyder HW, Intervention for fetal obstructive uropathy: has it been effective? Lancet 1987;: 1007-10.

6 Guys JM, Borella F, Monfort G. Ureteropelvic junction obstructions: prenata diagnosis and neonatal surgery in 47 cases. $\mathcal{F}$ Pediatr Surg 1988;23:156-8. 7 Harrison MR, Golbus MS, Filly RA, et al. Management of the fetus with congenital hydronephrosis. F Pediatr Surg 1982;17:728-40.

8 McFadyen IR. Obstruction of the fetal urinary tract: a role for surgical intervention in utero? $\mathrm{BrMed} \mathcal{F}$ 1984;288:459-62.

\title{
Bilateral fetal uropathy: What is the outlook?
}

\author{
R J Arthur, H C Irving, D F M Thomas, J K Watters
}

\section{Abstract}

Objective-To assess the morbidity and mortality associated with a prenatal diagnosis of bilateral fetal uropathy.

Design-Retrospective study.

Setting-Departments of radiology, paediatric surgery, obstetrics, and pathology in two teaching hospitals that serve as referral centres for the Yorkshire region.

Patients-126 Cases of fetal uropathy were referred either prenatally or postnatally from hospitals in Yorkshire between August 1982 and December 1987. The disease was bilateral in 54 cases and unilateral in 72 cases. In 14 cases bilateral fetal uropathy was associated with coexistent disease.

Interventions-All cases were managed individually by an obstetrician after discussion with the radiologists and paediatric surgeons. Babies who survived were treated prophylactically with antibiotics after delivery and were operated on if appropriate.

End point-Assessment of prognosis for long term renal function for each baby referred between August 1982 and December 1987: follow up ranged from six months to five years.

Measurements and main results-Of the 54 fetuses with bilateral fetal uropathy, 13 were terminated as the prenatal findings of ultrasonography were considered to be incompatible with long term survival. Ten of the liveborn babies died, five of renal or pulmonary insufficiency, or both, and five of associated congenital anomalies. Thirty one infants survived to follow up; four of these had serious coexistent disease and two had impaired renal function. Thus the overall mortality was $43 \%$ and the morbidity rate $19 \%$. The renal anomaly was associated with other serious disease in 14 cases (26\%) compared with two $(3 \%)$ of the 72 cases of unilateral fetal uropathy. All but two of the 27 infants with isolated bilateral urinary tract disease had excellent prospects for survival.

Conclusion-Although bilateral fetal uropathy is associated with a high morbidity rate and mortality, careful prenatal assessment can help to identify fetuses with a poor prognosis. The outlook for a fetus with isolated renal disease if treated promptly after delivery is excellent and compares favourably with that reported after prenatal surgical intervention.

\section{Introduction}

Detection of fetal urinary tract anomalies may be valuable for both prenatal and postnatal management, particularly when the abnormality is bilateral. ${ }^{1}$ The potential benefit is, however, offset by the poor prognosis often associated with bilateral fetal uropathy. Doctors had hoped that the introduction of fetal surgery would help to reduce the morbidity and mortality associated with this disease by preventing pulmonary hypoplasia and progressive impairment of renal function. Several recent studies have discussed the possible benefits of decompressing the fetal urinary tract surgically. ${ }^{2-4}$ Though the authors generally accepted that intervention is not justified for unilateral disease, they disagreed about the management of bilateral disease. We carried out a study to establish the morbidity and mortality associated with bilateral fetal uropathy, with particular reference to the incidence of coexistent congenital abnormalities and the long term outlook for renal function.

\section{Patients and methods}

Between August 1982 and December 1987, 126 cases of fetal uropathy were referred to our units either prenatally or postnatally for further assessment. The disease was bilateral in 54 cases; 28 of these were from our own districts, and 26 were referred from other hospitals throughout the Yorkshire region. Most of the anomalies had been detected by routine antenatal ultrasonography, which is normally performed at 17-19 weeks of gestation. There was no fixed policy for following these cases as generally we considered that each case should be managed individually and that the severity of the abnormality should be taken into consideration.

We reviewed the prenatal and postnatal records, including necropsy reports if appropriate, and assessed the incidence of congenital abnormalities in fetuses with unilateral and bilateral uropathy. Cases of equivocal dilatation of the urinary tract were omitted. Routine ultrasonography is performed on at least $95 \%$ of all pregnant women in Leeds, allowing us to estimate the overall incidence of fetal anomalies in the population.

\section{Results}

During the study 46775 pregnancies were registered in Leeds. We saw 78 fetuses with an important urinary tract anomaly; the disease was bilateral in 28 of these. This gave an incidence of all anomalies of the urinary tract of 1 in 600 pregnancies and an incidence of bilateral fetal uropathy of 1 in 1670 pregnancies.

Thirteen of the 54 fetuses with bilateral uropathy were terminated as the antenatal findings were incompatible with long term survival; eight had a primary urinary tract anomaly and in five the uropathy 